\title{
The iris naevus (Cogan-Reese) syndrome: light and electron microscopic observations
}

\author{
RALPH C. EAGLE, JR., RAMON L. FONT, \\ MYRON YANOFF, ${ }^{2}$ AND BEN S. FINE ${ }^{1}$ \\ From the ${ }^{1}$ Department of Ophthalmic Pathology, Armed Forces Institute of Pathology, \\ Washington, DC, and the ${ }^{2}$ Department of Ophthalmology, University of Pennsylvania and the \\ Scheie Eye Institute, Philadelphia, USA
}

SUMMARY The light and electron microscopic findings in an eye enucleated from a 38-year-old woman with iris naevus (Cogan-Reese) syndrome are presented. The clinical manifestations of this disease, namely, iridic stromal matting, iridic nodule formation, and unilateral angle-closure glaucoma, appear to be secondary to an endothelial membrane on the anterior surface of the iris. The iris naevus syndrome is now thought to represent one end of the clinical spectrum of an iridocorneal endothelial syndrome which also includes those clinical entities classified previously as Chandler's syndrome and essential iris atrophy.

The iris naevus (Cogan-Reese) syndrome is an entity characterised by unilateral glaucoma typically found in young to middle-aged women who develop anterior synechiae in a previously open angle. ${ }^{12}$ Iris abnormalities, including stromal matting with effacement of normal surface architecture, ectropion uveae, multiple pigmented iris nodules, and naevi of the anterior iridic surface are characteristic findings.

It has recently been suggested that the iris naevus syndrome, Chandler's syndrome, and essential iris atrophy represent the clinical spectrum of a single disease whose underlying pathogenetic mechanism is an abnormal proliferation of corneal endothelial cells leading to secondary iris abnormalities. The secondary iris manifestations seen clinically in this iridocorneal endothelial (ICE) syndrome are thought to result from variations in the pattern, rate, and extent of endothelial proliferation..$^{3-5}$ The light and electron microscopic findings reported here suggest that the iris alterations of the iris naevus syndrome are secondary to an endothelial membrane on its anterior surface.

The opinions or assertions contained herein are the private views of the authors and are not to be construed as official or as reflecting the views of the Department of the Army or the Department of Defense.

Correspondence to the Department of Ophthalmic Pathology, Armed Forces Institute of Pathology, Washington DC 20306, USA.

\section{Patient and methods}

\section{CASE REPORT}

A 35-year-old woman was first seen in April 1974 with a history of pain, pupillary irregularity, and heterochromia iridis in her left eye of several months' duration. The visual acuities were 20/20 OD and 20/50 OS. Ophthalmological examination revealed mild corneal oedema, ectropion uveae, and multiple small pigmented iris nodules in her left eye. The right eye appeared normal. The intraocular pressure was $16 \mathrm{mmHg}$ OD and 70 OS by applanation tonometry. The positive findings were confined to the left eye. Total peripheral anterior synechiae occluded the anterior chamber angle. The optic disc was deeply excavated. The visual field showed marked constriction.

No stigmata or family history of neurofibromatosis were found. The results of serum calcium and phosphorus and serum protein electrophoresis were normal. Chest $x$-ray showed no abnormalities. After a short unsuccessful trial of pilocarpine and acetazolamide a posterior lip sclerectomy with peripheral iridectomy was performed under a scleral flap. The intraocular pressure remained controlled without medication until December 1974. At this time the filtering bleb was smaller than previously noted and the intraocular pressure was $27 \mathrm{mmHg}$. Epinephrine and subsequently pilocarpine were started. In August 1975 the vision in her left eye 


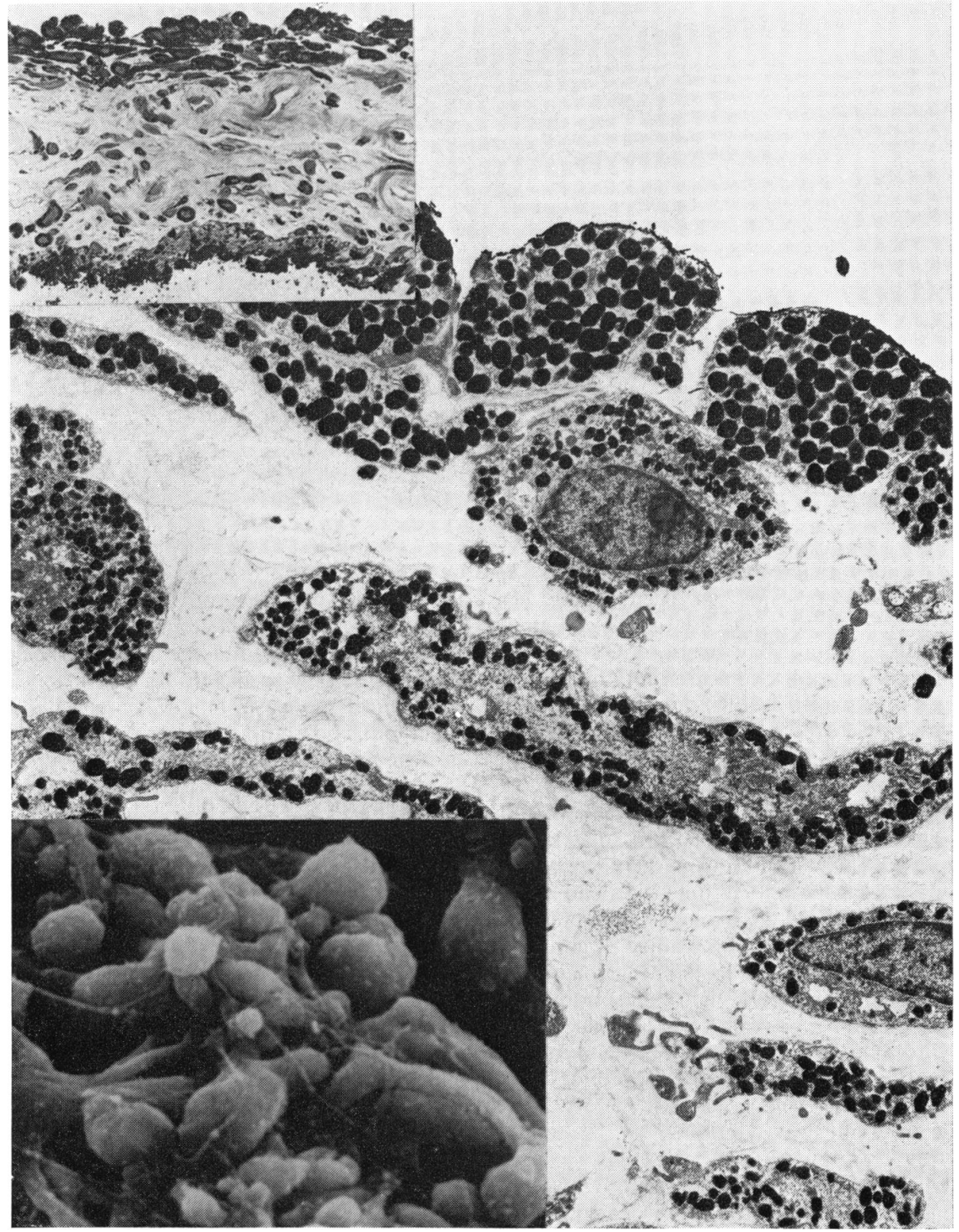

Fig. 1 Plump pigmented cells (in nonendothelialised portion of iris) containing myriad spheroidal melanin granules are present on the anterior iridic surface. The deeper stromal melanocytes show elongated cytoplasmic processes containing smaller, round to oval melanin granules. TEM, $\times 6810$. Upper inset: globoid, heavily pigmented cells on the anterior iridic surface. Paraphenylenediamine, $\times 325$. Lower inset: scanning electron micrograph from the same area discloses the round to plump configuration of the superficial naevoid cells. $\times 1540$.(AFIP neg. 79-13170-1) 
Fig. 2 Cohesive plaque of naevoid cells (iris nodule) rests on surface of thick endothelial basement membrane. A thin advancing sheet of melanocytes is present on the right. The basement membrane is interrupted (between arrows). Toluidine blue, $\times 630$. AFIP neg. $78-4670$

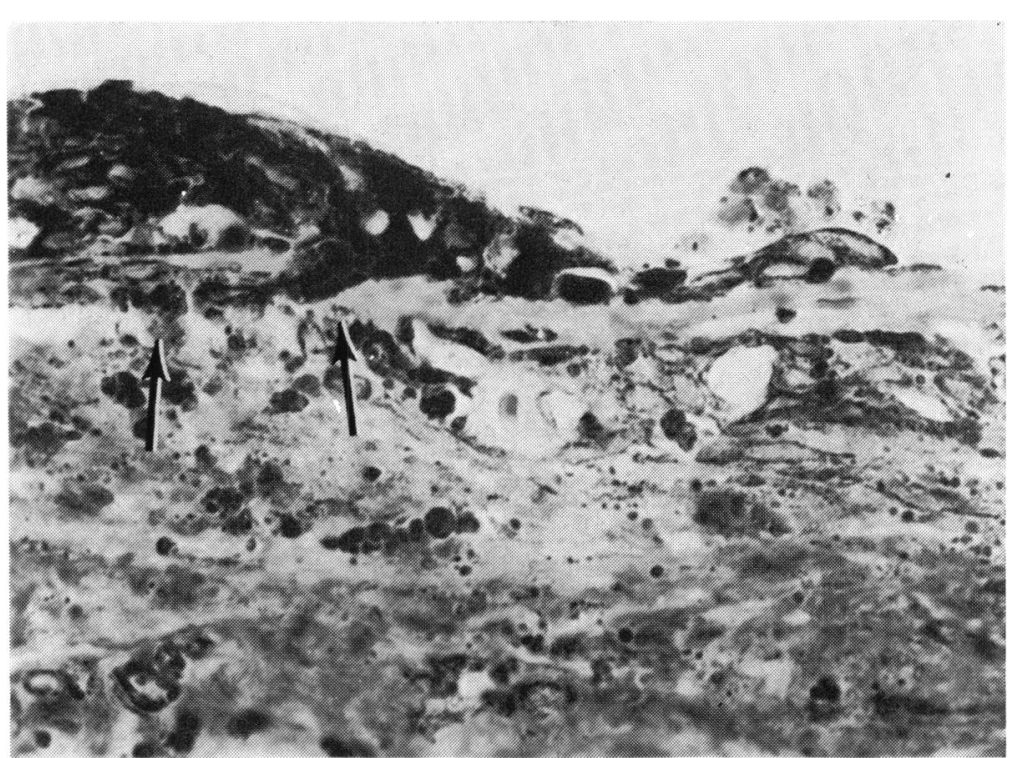

was $20 / 20$ and the intraocular pressure was 27 mmHg. The patient, who had been taking her medications sporadically, was not seen again until May 1976. Her intraocular pressure OS was 54 $\mathrm{mmHg}$, and the optic disc appeared totally cupped. Visual field evaluation could not be performed reliably. In July 1976 the filtering area appeared nonfunctioning. Maximal medical therapy with echothiophate iodide $0.125 \%$ and acetazolamide sequels failed to control the tension. One month later her visual acuity had dropped to 20/400. The patient refused further filtering surgery or cyclocryotherapy. In February 1977 the vision had dropped to hand motions, and the intraocular pressure was $50 \mathrm{mmHg}$. The patient complained of anorexia and paraesthesias secondary to the acetazolamide.

In July 1977 the vision was light perception. Two plus corneal oedema was present. The globe was quite hard to palpation. The patient rejected further glaucoma surgery or retrobulbar alcohol block and chose to have the left eye enucleated in July 1977. The patient's right eye continued to be totally normal.

The globe was fixed in a $10 \%$ neutral buffered formalin and submitted to the Department of Ophthalmic Pathology, Armed Forces Institute of Pathology, for histological examination.

\section{HISTOLOGICAL METHODS}

Routine paraffin-embedded sections for light microscopy were stained with haematoxylin and eosin ( $\mathrm{H}$ and $\mathrm{E})$ and periodic acid-Schiff (PAS). Bleached preparations also were prepared. Additional forma- lin-fixed wet tissue also was submitted for scanning and transmission electron microscopy (SEM and TEM). Thick sections (1-2 $\mu \mathrm{m})$ for light microscopy were stained with toluidine blue and paraphenylenediamine. The specimens for SEM were dried by the critical point technique, coated with goldpalladium, and examined in the AMR 1000 scanning electron microscope. After the completion of SEM examination a portion of the specimen was resubmitted for TEM.

\section{Results}

Macroscopically the irregular pupil showed marked ectropion uveae. A peripheral surgical coloboma of the iris was present at 12 o'clock. The limbal tissues had a slight bluish discoloration in the area of the filtering cicatrix. After vertical sectioning of the globe the chamber angle was seen to be closed by extensive peripheral anterior synechiae. Numerous small, dark brown to black nodules were present on the anterior surface of the light brown iris. The lens appeared cataractous. The optic disc was deeply cupped.

Microscopically the corneal endothelium was moderately atrophic, with focal areas of attenuation. Superiorly, adjacent to a surgical iris coloboma, the endothelium had extended into the filtering bleb, where it had laid down a new layer of abnormal basement membrane (ABM) material which resembled Descemet's membrane. The angle of the deep anterior chamber was occluded by peripheral anterior synechiae of varying width. Focally the 
adherent iris occluded only a portion of the filtering trabecular meshwork.

Central to the pseudoangle, endothelium and new ABM were present on the inner surface of the trabecular meshwork. Endothelium also had extended on to the anterior iridic surface, where it had elaborated a thin layer of ABM. The anterior surface of the moderately atrophic iris was flattened, with effacement of contraction furrows and crypts. The anterior iris was thickened focally by an increased number of benign-appearing melanocytic cells, which resembled naevus cells. One segment of the peripheral iris was not covered by an endothelial membrane. In this area a population of rather plump,

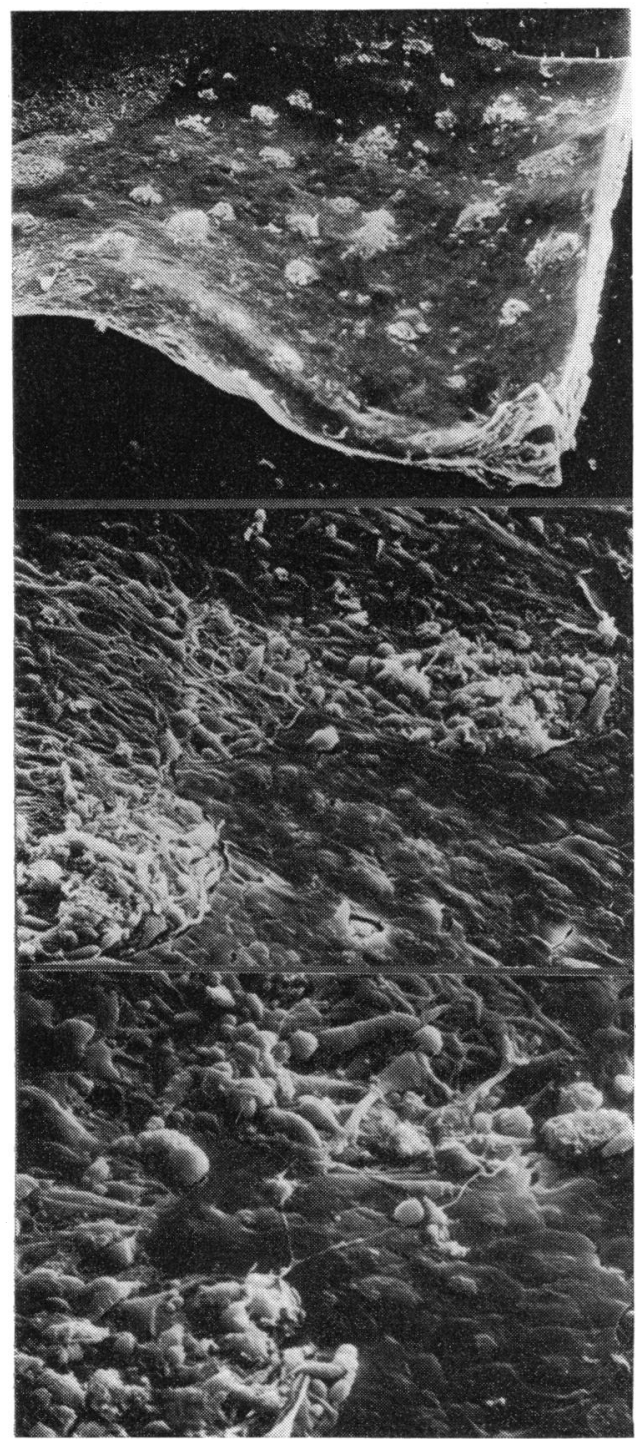

deeply pigmented melanocytic cells was present on the anterior iridic surface covering a layer of dendritic stromal melanocytes (Fig. 1, upper inset).

Numerous iris nodules, which were composed of pigmented cells, were found only in the endothelialised portion of the iris. These nodules were encircled by and partially rested on the surface of the newly elaborated ABM material (Fig. 2). Beneath the nodules the incomplete layer of $\mathrm{ABM}$ was considerably attenuated and almost devoid of endothelial cells. Continuity between the nodules and the underlying iridic stroma was maintained through a central aperture in the otherwise continuous sheet of ABM. Clumps of pigmented macrophages were noted on the surface of some iris nodules. Extensive ectropion of the iridic pigment epithelium and sphincter muscle was present. The lens nucleus showed mild, increased homogeneity, probably consistent with an early nuclear cataract. The retina showed extensive atrophy of its inner layers. The optic disc was deeply excavated and the optic nerve was markedly atrophic.

Scanning electron microscopy showed a continuous sheet of irregularly polygonal endothelial cells on the anterior iridic surface (Fig. 3). Covering the majority of the anterior iris, the membrane obscured the underlying stroma, effacing its normal pattern. The endothelial cells had scattered cilia and numerous microvilli, which were most prominent near intercellular junctions. Peripherally an island of iris stroma was not covered by endothelium. Here a population of plump to globular pigmented cells rested on the surface of the normal stromal melanocytes (Fig. 1, lower inset). In contrast to the stout processes of the latter cells, the heavily pigmented surface cells had long interweaving neurite-like processes.

Bordering the island of endothelial-free stroma, the advancing edge of the endothelial membrane had a scalloped configuration (Fig. 3, centre). Tongues of proliferating endothelium appeared to be surrounding or embracing aggregates of plump stromal cells of the iridic surface. Iris nodules were

Fig. 3 Top: Scanning electron microscopy of iris surface showing effacement of architecture by a sheet of endothelial cells. Multiple well-defined nodules of melanocytic cells are present only in the endothelialised portion of the iris. Relatively normal iris architecture is seen in the upper left. SEM, $\times 28$. Centre: Edge of advancing endothelial membrane displaying smoothly scalloped border and abrupt transition to relatively normal iris stroma. SEM, $\times 257$. Bottom: Advancing tongue of endothelial membrane on the right is composed of cells with irregularly polygonal outlines. SEM, $\times$ 550. AFIP neg. 79-13170-2 
Fig. 4 Nodule of melanocytic cells encircled by flat endothelial membrane with sharply defined margin (on the right). Plump melanocytic cells are evident on the surface of the nodule. SEM, $\times$ 1216. AFIP neg. 79-13170-3

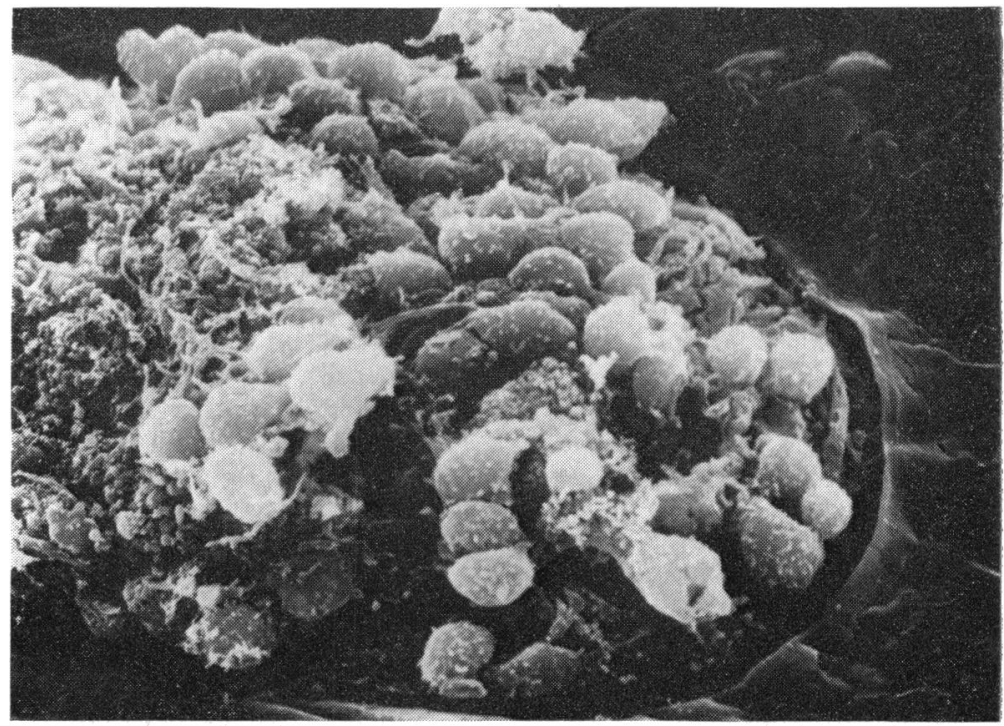

seen only in the endothelialised portion of the iris (Fig. 3 top and Fig. 4). Generally free of endothelial cells on their surface, the round to oval nodules measured approximately $1 / 10$ th of a millimetre in greatest diameter and were composed of two cell populations similar to those seen in the nonendothelialised island of stroma. Plump melanocytic cells were especially prominent on the surface of the nodules (Fig. 4). Occasionally the cells forming the nodules appeared to extend on to the surface of the encircling endothelial membrane.

Transmission electron microscopy confirmed the endothelial nature of the cells covering the anterior iridic surface (Fig. 5). Joined by tight junctions, the cells had elaborated copious quantities of AMB, which was predominantly filamentous. Patches of banded basement membrane (100 $\mathrm{nm}$ periodicity) also were present (Fig. 5, inset). Two distinct populations of melanocytic cells were observed in the iridic stroma. The heavily pigmented, plump surface cells contained melanin granules that were larger than those found in the underlying stromal melanocytes (Fig. 1). The size of these granules appeared intermediate between those of the normal uveal melanocytes and the iridic pigment epithelium. The iris nodules were composed of similar melanocytic cells. A significant number of these cells had large melanin granules.

\section{Discussion}

The constellation of anterior segment findings known as the iris naevus (Cogan-Reese) syndrome usually occurs in young to middle-aged women, who present commonly with unilateral secondary glaucoma and iris abnormalities. ${ }^{12}$ The syndrome is now thought to represent one end of the clinical spectrum of a disease that also includes Chandler's syndrome and essential iris atrophy. The basic pathogenetic event in this iridocorneal endothelial (ICE) syndrome is an abnormal proliferation of corneal endothelium that leads to trabecular obstruction, anterior synechia formation, and a spectrum of iris abnormalities, all of which are secondary to variations in the pattern, rate, and extent of endothelialisation..$^{3-6}$

Characteristic iris abnormalities in the iris naevus syndrome include effacement of the normal architecture of the iridic stroma, which appears matted and smudged; pupillary distortion with extensive ectropion uveae; diffuse naevi of the anterior stroma; and multiple pigmented iris nodules. ${ }^{12}$ The light and electron microscopic findings described herein suggest strongly that these iridic manifestations are indeed secondary to endothelial proliferation, a finding which had been noted repeatedly in previous light microscopic studies.

Stromal smudging and effacement of normal surface architecture, including the collarette, result from the presence of an anterior surface naevus and a continuous sheet of endothelial cells and abnormal basement membrane material on the anterior surface of the iris. Contraction of this transparent membrane presumably leads to pupillary distortion and ectropion uveae. The fibrovascular membrane of rubeosis iridis is not usually present and therefore cannot be considered the cause of the latter 2 findings. 


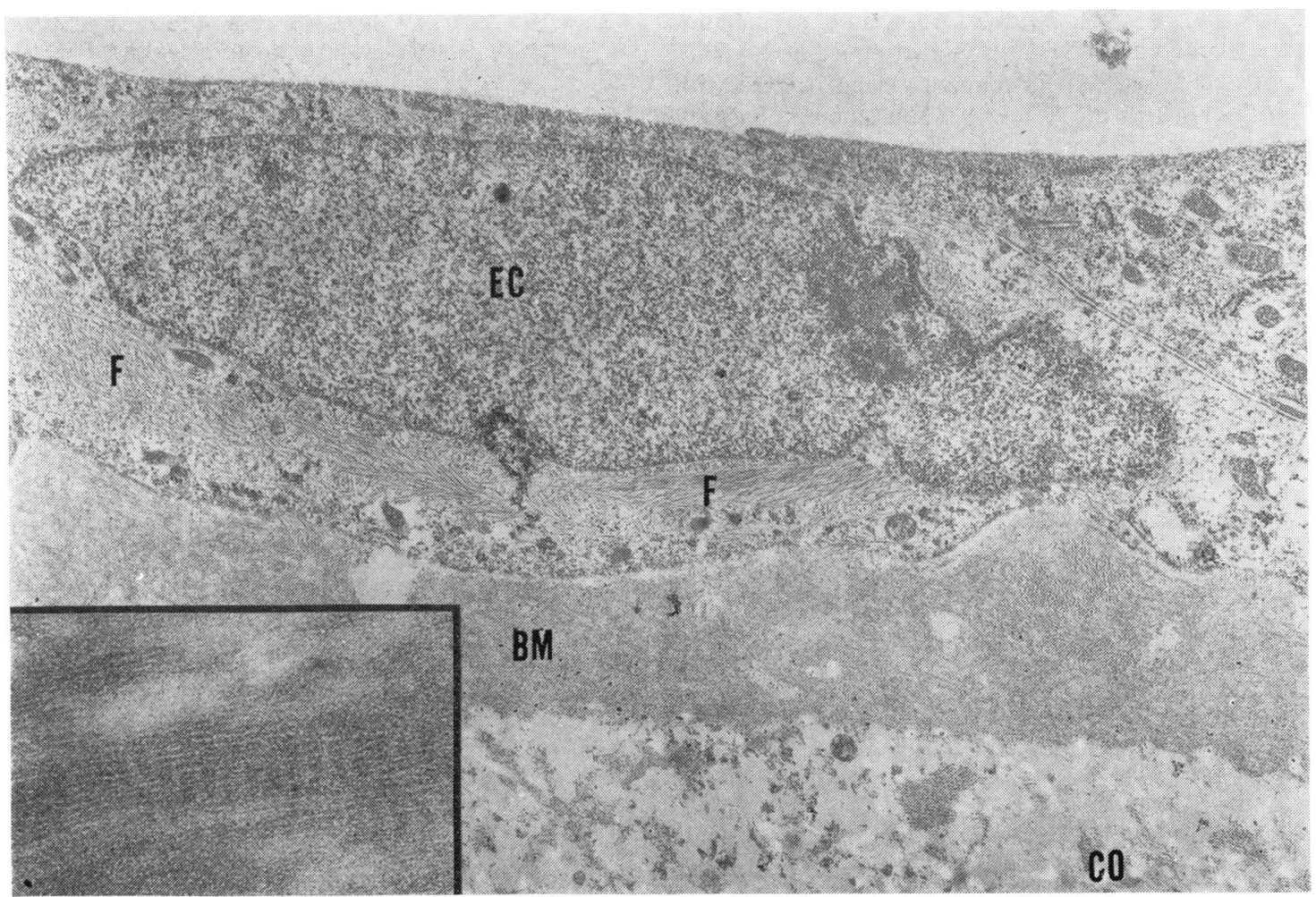

Fig. 5 Monolayer of endothelial cells $(E C)$ resting on the iridic surface has elaborated a thick basement membrane $(B M)$. CO: collagen $;(F):$ cytoplasmic filaments. TEM, $\times 13$ 145. Inset shows patch of filamentous banded basement membrane with a periodicity of $100 \mathrm{~nm}$. TEM, $\times 65300$. AFIP neg. 79-13170-4.

Endothelial proliferation probably also plays a major role in the formation of iris nodules, as originally suggested by Shields and coworkers. ${ }^{7}$ The confinement of nodules to the endothelialised portion of the iris, attenuation of endothelial ABM underlying the melanocytic nodules, and the presence of apertures within the basement membrane providing continuity with underlying stroma suggest that proliferating endothelium may encircle and pinch off portions of stroma. Contact inhibition between the advancing sheet of endothelium and collections of abnormal iridic melanocytes could facilitate stromal encirclement and is consistent with the scalloped border of the endothelial membrane seen by SEM. On serial clinical observation iris nodules have been noted to increase in size and pigmentation in essential iris atrophy. ${ }^{7}$ The extension of nodule-forming melanocytes on to the surface of adjacent ABM seen histologically suggests that these cells undergo active proliferation, though herniation of iridic stroma resulting from increasing contraction of the endothelial membrane is an alternative explanation.
Iris abnormalities usually do not occur when corneal endothelial overgrowth follows trauma or is found in association with rubeosis iridis caused by a variety of entities (for example, diabetes mellitus and central retinal vein thrombosis). This suggests that there is probably some sort of predetermined iris abnormality in the ICE syndrome. The term iris naevus syndrome was proposed by Scheie and Yanoff $^{2}$ to emphasise the diffuse, rarely full-thickness iridic naevi and iridic nodules composed of naevoid cells observed in those patients described originally by Cogan and Reese. ${ }^{1}$ The plump cells with larger melanin granules demonstrated on the anterior border layer in our case probably represent a population of atypical melanocytes, that is, naevuslike cells. Jakobeic et al. ${ }^{8}$ described 2 cases with early focal iridic endothelialisation in the vicinity of discrete full-thickness iris naevi and suggested that abnormal melanocytic cells may in some manner stimulate endothelial proliferation and overgrowth onto the iris. Naevi of the iris have been described clinically and pathologically in cases previously classified as essential iris atrophy. ${ }^{9-11}$ 
Furthermore, iridic nodules composed of melanocytic cells histologically indistinguishable from naevus cells are not infrequently seen in eyes with full-thickness iridic holes. ${ }^{57}$ It must be emphasised, however, that the naevus-like appearance seen histologically in some cases of essential iris atrophy and iris naevus syndrome could result from stromal compaction by the endothelial membrane. Further research is necessary to characterise the relationship, if any, between abnormal iridic melanocytes and the initiation of endothelial proliferation in the iridocorneal endothelial syndrome.

This study was supported in part by Research Grant EY00133 and in part by Training Grant EY-07040 from the National Eye Institute, National Institutes of Health, Bethesda, MD.

The authors thank Dr Martin L. Lipson for his detailed clinical summary. Technical assistance was provided by Efrain Perez-Rosario, James Stripling, and Sgt Walter Saddler, USAF.

\section{References}

${ }^{1}$ Cogan DG Reese AB. A syndrome of iris nodules, ectopic Descemet's membrane and unilateral glaucoma. Doc Ophthalmol 1969; 26: 424-33.
${ }^{2}$ Scheie HG, Yanoff M. Iris nevus (Cogan-Reese) syndrome: a cause of unilateral glaucoma. Arch Ophthalmol 1975; 93: 963-70.

${ }^{3}$ Eagle RC, Font RL, Yanoff M, Fine BS. (1978). The iridocorneal endothelial (ICE) syndrome: Primary proliferative endotheliopathy with secondary iris abnormalities. Presented at Southern section meeting, ARVO, Oklahoma City, OK, Sept. 9.

${ }^{4}$ Campbell DG, Shields MB Smith TR. The corneal endothelium and the spectrum of essential iris atrophy. Arch Ophthalmol 1978; 86: 317-24.

${ }^{5}$ Eagle RC, Font RL, Yanoff M, Fine BS. Proliferative endotheliopathy with iris abnormalities: the iridocorneal (ICE) syndrome. Arch Ophthalmol 1979; 97: 2104-11.

${ }^{6}$ Shields MB, Campbell DG, Simmons RJ. The essential iris atrophies. Am J Ophthalmol 1978; 85: 749-59.

'Shields MB, Campbell DG, Simmons RJ, Hutchinson BT. Iris nodules in essential iris atrophy. Arch Ophthalmol 1976: 94: 406-410.

${ }^{8}$ Jakobeic FA, Yanoff M, Mottow L, Anker P, Jones IS. Solitary iris nevus associated with peripheral anterior synechiae iris endothelialization. Am J Ophthalmol 1977; 183: 884-91.

${ }^{9}$ Lichter PR. The spectrum of Chandler's syndrome: an often overlooked cause of unilateral glaucoma. Trans Am Acad Ophthalmol Otolaryngol 1978; 85: 245-51.

${ }^{10}$ Sugar HS. Glaucoma and essential progressive atrophy of the iris. Am J Ophthalmol 1945; 28: 744-48.

${ }^{11}$ Rochat FG, Mulder W. On progressive atrophy of the iris with formation of holes and glaucoma. $\mathrm{Br} J$ Ophthalmol $1918 ; 8: 362-6$. 